\title{
Allergic contact dermatitis caused by (meth)acrylates in nail cosmetic products in users and nail technicians - a 5-year study
}

\begin{abstract}
Inês Raposo ${ }^{1}$, Inês Lobo ${ }^{1}$, Cristina Amaro², Maria de Lurdes Lobo², Helena Melo ${ }^{3}$, Joana Parente ${ }^{4}$, Teresa Pereira ${ }^{5}$, Joana Rocha ${ }^{6}$, Ana P. Cunha ${ }^{7}$, Armando Baptista ${ }^{8}$, Pedro Serrano ${ }^{9}$, Teresa Correia ${ }^{10}$, Ana R. Travassos ${ }^{3,10}$, Margarida Dias ${ }^{11}$, Fátima Pereira ${ }^{12}$ and Margarida Gonçalo ${ }^{13}$

${ }^{1}$ Department of Dermatology, Centro Hospitalar do Porto, 4099-001 Porto, Portugal, ${ }^{2}$ Department of Dermatology, Centro Hospitalar Lisboa Central, 1169-050 Lisboa, Portugal, ${ }^{3}$ Department of Dermatology, Hospital CUF Descobertas, 1998-018 Lisboa, Portugal, ${ }^{4}$ Department of Dermatology, Hospital de Santarém, 2005-177 Santarém, Portugal, ${ }^{5}$ Department of Dermatology, Hospital de Braga, 4710 Braga, Portugal, ${ }^{6}$ Department of Dermatology, Hospital Pedro Hispano, 4460 Matosinhos, Portugal, ${ }^{7}$ Department of Dermatology, Centro Hospitalar São João, 4200-319 Porto, Portugal, ${ }^{8}$ Department of Dermatology, Centro Hospitalar Vila Nova de Gaia, 4400-129 Vila Nova de Gaia, Portugal, ${ }^{9}$ Department of Dermatology, Hospital Garcia da Orta, $2801-951$ Almada, Portugal, ${ }^{10}$ Department of Dermatology, Hospital de Santa Maria, Centro Hospitalar Lisboa Norte, 1649-035 Lisboa, Portugal, ${ }^{11}$ Department of Dermatology, Hospital Egas Moniz, Centro Hospitalar Lisboa Ocidental, 1349-019 Lisboa, Portugal, ${ }^{12}$ Department of Dermatology, Hospital CUF Infante Santo, 1350-070 Lisboa, Portugal, and ${ }^{13}$ Clinic of Dermatology, University Hospital and Faculty of Medicine, University of Coimbra, 3000-075 Coimbra, Portugal

doi:10.1111/cod.12817
\end{abstract}

\section{Summary}

Background. The increasing use of long-lasting nail aesthetic products has led to a growing number of cases of allergic contact dermatitis (ACD) caused by (meth)acrylates in recent years.

Objectives. To provide information on ACD caused by (meth)acrylates related to nail cosmetic products.

Methods. We retrospectively reviewed files of patients with ACD caused by (meth)acrylates related to nail cosmetic products, who were patch tested between January 2011 and December 2015 in 13 departments of dermatology in Portugal.

Results. Two-hundred and thirty cases of ACD caused by (meth)acrylates ( 55 technicians, 56 consumers, and 119 with mixed exposure) had been documented, mostly as chronic hand eczema (93\%). The most common sensitizers were: 2-hydroxyethyl methacrylate (HEMA), which was positive in $90 \%$ of the tested patients, 2-hydroxypropyl methacrylate (HPMA), which was positive in $64.1 \%$, and ethyleneglycol dimethacrylate, which was positive in $54.5 \%$.

Conclusion. HEMA and HPMA were the most frequent positive allergens. HEMA, which identified $90 \%$ of cases, can be considered to be a good screening allergen. The high number of cases of ACD caused by (meth)acrylates in nail cosmetic products certainly warrants better preventive measures at the occupational level, and specific regulation in the field of consumer safety.

Key words: acrylates; allergic contact dermatitis; artificial nails; methacrylates; occupational.

Acrylates and methacrylates [summarized as (meth)acrylates in the following] are chemical derivatives

Correspondence: Inês Raposo, Serviço de Dermatologia, Centro Hospitalar do Porto, Edifício das Consultas Externas, Ex-CICAP, Rua D. Manuel II, s/n, 4099-001 Porto, Portugal. Tel: +351226097429; Fax: +351226097429. E-mail: inesraposovs@gmail.com

Conflicts of interest: The authors declare no conflict of interest.

Accepted for publication 21 March 2017 of (meth)acrylic acid, and yield acrylic plastics after polymerization. (Meth)acrylates are used in a wide range of occupational settings as glues, coatings, and plastic materials, both in industrial work and in dental restorative work $(1,2)$. Although acrylic plastic polymers normally do not cause contact allergy, their monomeric and dimeric forms have high sensitizing potency, and thus can cause allergic contact dermatitis (ACD) in such occupational settings $(1,3)$. Given the relevance of this problem, 
recommendations for adequate personal protection and the development of 'non-touch' techniques have been implemented in the past years, in order to reduce the allergic burden of (meth)acrylates, particularly in the industrial and dentistry fields.

More recently, the popularity and growing use of nails made from (meth)acrylates and long-lasting acrylic nail lacquer have created another source of occupational and consumer exposure to (meth)acrylates, and an increase in the frequency of work-related ACD in exposed nail technicians (beauticians) has been identified (4-7). Nail aesthetics are subdivided into: (i) acrylic nails formed from a mixture of powder and liquid (meth)acrylates, which polymerize; (ii) photobonded/gel nails, made by applying acrylate gel over the nail plate alone or with the addition of a tip to extend the nail plate, and then polymerizing under ultraviolet (UV) light; (iii) press-on nails; and (iv) 'silk nails.' The last two of these represent pre-formed nails that are glued onto natural nails (8). Acrylic and gel nails do not polymerize completely after mixing, with or without hardening with UV light, and monomers are therefore still present when nails are sculpted (9). Pre-formed nails (press-on and 'silk nails') do not contain (meth)acrylate monomers, and their use does not cause ACD caused by these allergens. However, the chemical compound present in almost every glue that is used to attach pre-formed nails - ethyl cyanoacrylate - has been reported as an allergen in this setting (8). Although cyanoacrylates do not cross-react with (meth)acrylates, they may be independently responsible for sensitization, and represent another trigger of ACD in artificial nail technicians and users $(8,10,11)$.

Given the importance of this subject, the aim of our study was to identify and characterize cases of ACD caused by (meth)acrylates in users and technicians of nail aesthetics in 13 Portuguese departments of dermatology during a 5-year period.

\section{Materials and Methods}

We retrospectively reviewed the files of patients with ACD caused by (meth)acrylates in relation to nail aesthetics, who were patch tested between January 2011 and December 2015 in 13 departments of occupational and environmental dermatology in Portugal. All patients were patch tested with the Portuguese and European baseline series and an extended series of 15-17 (meth)acrylates (Chemotechnique Diagnostics, Vellinge, Sweden). The indication for patch testing was based on the presence of eczema in users or technicians exposed to nail cosmetic products. The allergens were placed in Finn Chambers ${ }^{\circledR}$ on Scanpor ${ }^{\circledR}$ tape (20 mg in 8-mm chambers), and immediately applied to the patient's upper back. Allergens were left in place for $48 \mathrm{~h}$, and readings were performed on day (D) 3 for all patients. Patients were instructed to return on D7 if late additional reactions were observed, which is a common practice to reduce false-negative readings in our Portuguese network. Scoring of positive reactions comprised weak $(+)$, strong $(++)$ and extreme $(+++)$ positive reaction grades according to ICDRG and ESCD recommendations (12). Demographic and clinical profiles of all patients were collected according to the MOAHLFA index (Male, Occupational, Atopy, Hand, Leg, Face, Age $\geq 40$ years).

\section{Results}

During the study period, among a total of 11639 patients patch tested in the 13 departments, $230(1.97 \%)$ cases of ACD caused by (meth)acrylates were detected, and classified as clinically relevant. Unfortunately, the number of patients tested with the (meth)acrylates series could not be exactly determined with the case series design used. Regarding the source of exposure, $23.9 \%(n=55)$ were occupationally exposed, $24.4 \%(n=56)$ were consumers, and $51.7 \%(\mathrm{n}=119)$ were exposed both as consumers and occupationally. The mean age of the patients was 36.9 years (age range 20-65 years), and all patients were females. A background of personal atopy or atopy in the family was identified in 77 patients (33.5\%). The most common presentation was chronic hand eczema $(93 \%, \mathrm{n}=214)$, mainly as pulpitis with fissures $(77.8 \%$, $\mathrm{n}=179$ ). Nail dystrophy affected 48 patients $(20.9 \%)$, namely, 2 with occupational exposure, 15 consumers, and 31 with mixed exposure. Ectopic lesions on the face were detected in 81 patients $(35.2 \%)$, namely, 15 with exclusively occupational exposure, 18 consumers, and 48 with mixed exposure. In nail technicians, the average time before symptoms developed after the technicians had started to work in the profession was 24.9 months (range 1 month to 8 years).

The most common sensitizers among the 230 allergic individuals were: 2-hydroxyethyl methacrylate (HEMA), which was positive in 198 cases $(90 \%$ of 220 patch tested patients), 2-hydroxypropyl methacrylate (HPMA), which was positive in 120 patients $(64.1 \%$ of 187 tested patients), and ethyleneglycol dimethacrylate (EGDMA), which was positive in 121 patients $(54.5 \%$ of 222 tested patients). Positive patch test reactions to ethyl cyanoacrylate were observed in 13 patients, of whom all reacted to other meth(acrylates) (9 reacted to both HEMA and HPMA, 3 reacted to HEMA, and 1 reacted to EGDMA).

Of the 22 patients who did not react to HEMA, 7 reacted to HPMA. The combination of these two allergens (HEMA + HPMA) identified a total of 
93.4\% of our patients, and the addition of EGDMA (HEMA + HPMA + EGDMA) identified 96.8\%. The combination of (meth)acrylates that identified all patients in our series was: HEMA, HPMA, EGDMA, triethylene glycol dimethacrylate, methyl methacrylate, ethyl methacrylate, ethyl acrylate, and hydroxyethyl acrylate.

\section{Discussion}

This study with 230 cases of ACD caused by (meth)acrylates used in nail cosmetic products represents one of the largest series concerning this problem, highlighting its increasing importance in Portugal, as in other European countries $(11,13)$. Other studies from Portugal have shown that nail technicians now represent $80 \%$ of occupational cases of ACD caused by (meth)acrylates (13), and are the subgroup affected most by ACD (7), reflecting an emerging profession-related health problem.

ACD caused by (meth)acrylates in nail cosmetic products usually manifests as hand eczema, especially with periungual involvement. Although less common, onycholysis, onychodystrophy and paraesthesia may occur (3, 8, 14, 15). Ectopic lesions are frequently reported, especially in nail artists, who apply the products on a professional basis. Specifically, facial lesions may result from airborne exposure to dust generated by nail sculpting or by evaporation of (meth)acrylate monomers during the procedures, and also by transfer from contaminated hands $(3,5,8,16,17)$. Eczema on the wrists and forearms can also occur, for example because of contact with contaminated working surfaces or tools (13).

Previous studies aimed to establish a screening patch test series that would allow the identification of the majority of cases of ACD caused by (meth)acrylates (8, $11,13,17-20)$. In our study, HEMA and HPMA were the most frequent positive allergens and, in agreement with prior studies, HEMA represents a good screening allergen, identifying $90 \%$ of allergic patients in our series. The screening sensitivity can be enhanced by the addition of HPMA to HEMA (93.4\%) and of EGDMA to HEMA + HPMA (96.8\%). Testing with a limited number of allergens instead of a whole (meth)acrylate series may prove to be important in order to avoid the numerous (strong/extreme) positive reactions frequently observed in this setting, and to minimize the risk of active sensitization (13). The authors consider the frequency of ACD caused by (meth)acrylates observed in our study to provide relevant support for the inclusion of HEMA in the Portuguese baseline series, in view of the good screening sensitivity and the growing use of (meth)acrylates in personal and occupational settings. Moreover, the fact that ectopic lesions sometimes constitute the only cutaneous presentation makes initial suspicion of (meth)acrylate contact allergy difficult, and favours routine application (21).

In addition, the patch test concentration of the allergens is important, as too high a concentration may lead to active sensitization, and too low a concentration may lead to false-negative results. The concentrations generally established as safe are $0.1 \%, 2 \%$ and $10 \%$ pet. for acrylates, methacrylates, and cyanoacrylates, respectively (1). For allergen storage and the application of patches, the high reactivity and volatility, respectively, need to be considered. Following their removal from the freezer, allergens should be immediately applied to the patient's back to prevent evaporation from the test chambers, which is a possible cause of false-negative results $(1,19,22)$.

Full product labelling to identify products that can be used by the patient is of limited help in this setting, as each product usually contains multiple acrylic contaminants that are not identified on the product package in addition to the main (meth)acrylate ingredients. Moreover, cross-reactivity between different (meth)acrylates may occur, limiting the usefulness of recommendations of theoretically safe products (8). Furthermore, all patients who reacted to ethyl cyanoacrylate also reacted to (meth)acrylates in our series. The latter represent patients who were previously sensitized to (meth)acrylates by regular application of gel/acrylic nails, and who later altered their artificial nail type to pre-formed products (press-on or 'silk nails'). This fact may indicate that, although pre-formed nails themselves can be considered to be non-sensitizing, other allergens present in the glue, such as ethyl cyanoacrylate, may cause ACD. Ethyl cyanoacrylate is another trigger of ACD in this occupational area, highlighting the need for caution when counselling patients about alternative products, namely pre-formed nails.

Given the facts discussed above, the best way to prevent sensitization is to implement personal safety measures, preferably early in the course of training, in order to reduce occupational exposure $(4,8)$. Latex, vinyl and nitrile gloves are rapidly permeated by (meth)acrylate monomers, and are thus inefficient as a sole protection measure, and would require frequent replacement (23). The $4 \mathrm{H}^{\circledR}$ (ethylene-vinyl alcohol-polyethylene) gloves offer the most effective protection. However, these gloves are bulky and inelastic, and impair dexterity (24), unless only the fingertips of the gloves are used below classic latex or vinyl gloves. A recent publication has shown that certain gloves, namely some nitrile gloves, can prevent (meth)acrylate permeation and the elicitation of positive patch test reactions for periods as long as $60 \mathrm{~min}$, which is sufficient for work on one single client (25). The use 
of 'non-touch' techniques, keeping occupational object surfaces clear from residues and frequently changing disposable gloves should generally be advised $(8,13,17)$.

The growing use of home kits designed for non-professional use is another target for establishing personal safety measures for consumers (26).

Our study has some limitations, given its retrospective nature, namely that not all patients were tested with the entire (meth)acrylate series (for example, $4.3 \%$ were not tested with HEMA), for instance because of a lack of allergen supply in a given department for a period, or deliberate omission because of positivity to other (meth)acrylates. Moreover, the retrospective collection of data in our study limited the analysis of the exact proportion of patients identified by late readings (D7), limiting the conclusions that can be drawn regarding the percentage of false-negative readings on D3. As data were collected only during certain periods within the actual study interval in some centres, time trends of sensitization during this period could not be addressed, and nor could the proportion of positive patients among all tested patients be determined.

Nonetheless, the authors would like to highlight the high number of cases of ACD caused by (meth)acrylates related to cosmetic nail products, both occupationally and in consumers. This, together with other reports of such data, should alert authorities to take regulatory measures concerning the safety of consumers, and to promote educational and preventive measures among nail technicians and consumers.

\section{References}

1 Sasseville D. Acrylates in contact dermatitis. Dermatitis 2012: 23: 6-16.

2 Sanchez-Perez J, Gonzalez-Arriba A, Goiriz R, Garcia-Diez A. Occupational allergic contact dermatitis to acrylates and methacrylates. Contact Dermatitis 2008: 58: 252-254.

3 Geukens S, Goossens A. Occupational contact allergy to (meth)acrylates. Contact Dermatitis 2001: 44: 153-159.

4 Uter W, Geier J. Contact allergy to acrylates and methacrylates in consumers and nail artists - data of the Information Network of Departments of Dermatology, 2004-2013. Contact Dermatitis 2015: 72: 224-228.

5 Kwok C, Money A, Carder M et al. Cases of occupational dermatitis and asthma in beauticians that were reported to The Health and Occupation Research (THOR) network from 1996 to 2011. Clin Exp Dermatol 2014: 39: 590-595.

6 Le Q, Cahill J, Palmer-Le A, Nixon R. The rising trend in allergic contact dermatitis to acrylic nail products. Australas J Dermatol 2015: 56: 221-223.

7 Pestana C, Gomes R, Pinheiro V et al. Main causes of occupational allergic contact dermatitis: a three year study in the Center of Portugal. Acta Med Port 2016: 29: 449-455.

8 Constandt L, Hecke E V, Naeyaert J M, Goossens A. Screening for contact allergy to artificial nails. Contact Dermatitis 2005: 52: $73-77$.

9 Kanerva L, Lauerma A, Jolanki R, Estlander T. Methyl acrylate: a new sensitizer in nail lacquer. Contact Dermatitis 1995: 33: 203-204.

10 Kanerva L, Jolanki R, Estlander T. 10 years of patch testing with the (meth)acrylate series. Contact Dermatitis 1997: 37: 255-258.

11 Goon A, Bruze M, Zimerson E et al. Contact allergy to acrylates/ methacrylates in the acrylate and nail acrylics series in southern Sweden: simultaneous positive patch test reaction patterns and possible screening allergens. Contact Dermatitis 2007: 57: 21-27.

12 Johansen J D, Aalto-Korte K, Agner T et al. European Society of Contact Dermatitis guideline for diagnostic patch testing - recommendations on best practice. Contact Dermatitis 2015: 73: 195-221.

13 Ramos L, Cabral R, Gonçalo M. Allergic contact dermatitis caused by acrylates and methacrylates - a 7-year study. Contact Dermatitis 2014: 71: 102-107.

14 Slodownik D, Williams J D, Tate B J. Prolonged paresthesia due to sculptured acrylic nails. Contact Dermatitis 2007: 56: 298-299.

15 Kieć-Świerczyńska M, Kręcisz B, Świerczyńska-Machura D, Zaremba J. An epidemic of occupational contact dermatitis from an acrylic glue. Contact Dermatitis 2005: 52: 121-125.

16 Fitzgerald D A, English J S. Widespread contact dermatitis from sculptured nails. Contact Dermatitis 1994: 30: 118.

17 Lazarov A. Sensitization to acrylates is a common adverse reaction to artificial fingernails. J Eur Acad Dermatol Venereol 2007: 21: 169-174.

18 Goon A T, Bruze M, Zimerson E et al. Screening for acrylate/methacrylate allergy in the baseline series: our experience in Sweden and Singapore. Contact Dermatitis 2008: 59: 307-313.
19 Christoffers W A, Coenraads P J, Schuttelaar M L. Two decades of occupational (meth)acrylate patch test results and focus on isobornyl acrylate. Contact Dermatitis 2013: 69: 86-92.

20 Sood A, Taylor J S. Acrylic reactions: a review of 56 cases. Contact Dermatitis 2003: 48: 346-347.

21 Montgomery R, Stocks S J, Wilkinson S M. Contact allergy resulting from the use of acrylate nails is increasing in both users and those who are occupationally exposed. Contact Dermatitis 2016: 74: 120-122.

22 Goon A T, Bruze M, Zimerson E et al. Variation in allergen content over time of acrylates/methacrylates in patch test preparations. Br J Dermatol 2011: 164: 116-124.

23 Pegum J S, Medhurst F A. Contact dermatitis from penetration of rubber gloves by acrylic monomer. $\mathrm{Br}$ Med J 1971: 2: 141-143.

24 Andersson T, Bruze M, Björkner B. In vivo testing of the protection of gloves against acrylates in dentin-bonding systems on patients with known contact allergy to acrylates. Contact Dermatitis 1999: 41: 254-259.

25 Ursberg A M, Bergendorff O, Thorsson A $\mathrm{C}$, Isaksson $\mathrm{M}$. Is there a good in vivo method to show whether gloves are sufficiently protective when a nail technician is exposed to (meth)acrylates? An in vivo pilot study. Contact Dermatitis 2016: 75: 62-65.

26 Dahlin J, Berne B, Duner K et al. Several cases of undesirable effects caused by methacrylate ultraviolet-curing nail polish for non-professional use. Contact Dermatitis 2016: 75: 151-156. 